\title{
The Relationship Between Physical Examination and Ultrasonography of Large Entheses of the Achilles Tendon and Patellar Tendon Origin
}

\author{
Sibel Zehra Aydin (1D, Sibel Bakirci, Esen Kasapoglu, Concepcion Castillo-Gallego, \\ Fatıma Arslan Alhussain, Zoe R. Ash, Esra Kurum, Dennis McGonagle, Helena Marzo-Ortega, \\ Dafna Gladman ${ }^{D}$, and Lihi Eder
}

\begin{abstract}
Objective. To investigate the relationship between physical examination (PE) and sonographic features of enthesitis, based on anatomical sites.

Methods. The analysis was done using merged raw data of 3 studies on 2298 entheses.

Results. Patients with clinical Achilles enthesitis had more abnormalities on ultrasound (US): hypoechogenicity, $\mathrm{p}<0.001$; thickening, $\mathrm{p}=0.001$; Doppler signals, $\mathrm{p}=0.002$; and erosions, $\mathrm{p}=0.02$. The patellar tendon origin also correlated with PE but distal patellar tendon insertion and plantar aponeurosis were uncoupled from the US.

Conclusion. The relationship between clinical and sonographic findings for large entheses is dependent on the anatomical site. For the patellar tendon origin and Achilles entheses, PE is significantly linked to US findings. (First Release March 1 2020; J Rheumatol 2020;47:1026-30; doi:10.3899/jrheum.190169)
\end{abstract}

Key Indexing Terms:

ULTRASOUND

PHYSICAL EXAMINATION

ENTHESOPATHY

From the University of Ottawa Faculty of Medicine, Ottawa Hospital Research Institute, Department of Internal Medicine, Division of Rheumatology, Ottawa, Ontario, Canada; Istanbul Medeniyet University, Faculty of Medicine, Department of Internal Medicine, Division of Rheumatology, Istanbul, Turkey; Complejo Hospitalario Torrecárdenas, Department of Internal Medicine, Division of Rheumatology, Almería, Spain; UK National Institute for Health Research (NIHR) Leeds Biomedical Research Centre, Leeds Teaching Hospitals Trust and Leeds Institute of Rheumatic and Musculoskeletal Medicine, University of Leeds, Leeds, UK; University of California, Department of Statistics, Riverside, California, USA; University of Toronto, Department of Internal Medicine, Division of Rheumatology, Krembil Research Institute, Toronto Western Hospital; University of Toronto, Women's College Research Institute, Women's College Hospital, Rheumatology, Toronto, Ontario, Canada.

This research is supported by the NIHR Leeds Biomedical Research Centre. The views expressed are those of the author(s) and not necessarily those of the UK National Health Service, the NIHR, or the UK Department of Health. S.Z.Aydin, MD, Assoc. Professor, University of Ottawa Faculty of Medicine, Ottawa Hospital Research Institute, Department of Internal Medicine, Division of Rheumatology; S. Bakirci, MD, University of Ottawa Faculty of Medicine, Department of Internal Medicine, Division of Rheumatology; E. Kasapoglu, MD, Assoc. Professor, Istanbul Medeniyet University, Faculty of Medicine, Department of Internal Medicine, Division of Rheumatology; C. Castillo-Gallego, MD, Complejo Hospitalario Torrecárdenas, Department of Internal Medicine, Division of Rheumatology; F. Arslan Alhussain, MD, Istanbul Medeniyet University, Department of Internal Medicine; Z.R. Ash, MD, NIHR Leeds Biomedical Research Centre, Leeds Teaching Hospitals Trust and Leeds Institute of Rheumatic and Musculoskeletal Medicine, University of Leeds; E. Kurum, PhD, Assistant Professor, University of California, Department of Statistics; D. McGonagle, MD, Professor, NIHR Leeds Biomedical Research Centre, Leeds Teaching Hospitals Trust and Leeds Institute of Rheumatic and Musculoskeletal Medicine, University of Leeds; H. Marzo-Ortega, MD, NIHR Leeds Biomedical Research Centre, Leeds Teaching Hospitals Trust and Leeds Institute of Rheumatic and Musculoskeletal Medicine, University of Leeds; D. Gladman, MD, Professor of Medicine, University of Toronto, Department of Internal Medicine, Division of Rheumatology, Krembil Research Institute, Toronto
Western Hospital; L. Eder, MD, PhD, Assistant Professor, University of Toronto, Women's College Research Institute, Women's College Hospital, Rheumatology.

Address correspondence to Dr. S.Z. Aydin, 1967 Riverside Drive, Ottawa, Ontario K1H 7W9, Canada.E-mail: saydin@toh.ca

Accepted for publication August 22, 2019.

Enthesitis is a characteristic sign and hallmark of spondyloarthritis (SpA) and is clinically defined as pain or tenderness at the attachment site of a tendon/ligament to the bone, with or without swelling. However, physical examination (PE) is neither sensitive nor specific for the evaluation of enthesitis $^{1}$. Ultrasonography (US) has been increasingly used for the assessment of enthesitis because it has the advantage of visualizing both soft tissue and bony changes ${ }^{2,3}$. Comparison between US with PE has mostly been reported in relation to the summation of total US scores with overall PE scores from multiple entheseal sites, rather than elementary lesions of enthesitis on US and per site ${ }^{4,5,6}$. US is usually considered more sensitive than PE in detecting enthesitis. However, it is also true that patients with clinical entheseal tenderness sometimes have no US feature of enthesitis ${ }^{7,8}$. Unlike synovitis, it is not feasible to evaluate and validate sonographic or clinical enthesitis against the gold standard of tissue biopsy, so the relevance of clinical and imaging findings for enthesitis is difficult to disentangle.

Our hypothesis is that PE may be overrating enthesitis at certain sites, whereas the link between US and PE can be better in others. There is limited information on the 
relationship between clinical and imaging findings from individual US lesions on multiple entheseal sites.

\section{MATERIALS AND METHODS}

The raw data of 3 previous studies were used for this analysis ${ }^{8,9,10}$. All 3 studies were approved by 3 different ethics boards [Marmara University Ethics Board, No. 09.2014.0143; Leeds (East) REC 09/H1306/105; The University Health Network, REB\# 08-0126-AE]. The Marmara University Ethics Board declared that no additional approval was required. The first study, by Aydin, et al, comparing the entheseal differences in psoriatic arthritis (PsA), psoriasis, and healthy controls ${ }^{8}$, had 1 sonographer (SZA), and $\mathrm{PE}$ was performed by 1 investigator (ZRA) on the same day, blinded to each other's assessment. A Logiq-E9 (General Electric) was used to scan 12 entheses: quadriceps insertion, patellar tendon origin and insertion, Achilles tendon and plantar aponeurosis insertions, and lateral epicondyle for the common extensor tendon origin, bilaterally. The elementary lesions defined by the Outcome Measures in Rheumatology (OMERACT) group have been used: hypoechogenicity, thickening, entheseal PD signals, erosions, enthesophytes, and calcifications ${ }^{11}$. All findings were graded $0-3$, quantitatively for thickening and erosions and semiquantitatively for the other lesions to determine lesion severity on US ${ }^{8}$.

For the second study, by Arslan Alhussain, et al, comparing the differences between PsA and ankylosing spondylitis ${ }^{9}$, the sonographer was the same (SZA), and used the same methodology as the aforementioned study, except that the US machine was a MyLab70-XVG (Esaote), with a 6-18 Mhz linear transducer. One clinician performed the PE of the entheses on the same day as the US (FAA), and that clinician and the sonographer were blinded to each other's assessment. For these 2 studies, only psoriasis or PsA data were extracted.

The third study, by Polachek, et al, examined the association between sonographic enthesitis and the severity of radiographic features of damage in the peripheral and axial joints in patients with PsA ${ }^{10}$. The US scans were done by 1 sonographer (LE) using a MyLab70-XVG equipped with a 6-18 $\mathrm{MHz}$ linear transducer. Clinical assessment of the entheses was performed at the same day by the rheumatologist evaluating the patient. The Madrid Sonographic Enthesitis Index scoring system was used in this study ${ }^{12}$. Therefore, in addition, the triceps tendon insertion was also scanned; however, this site was not analyzed because it was not included in the previous studies. The same elementary lesions were investigated. There were some differences for the scoring of the severity of the lesions: Doppler signals and erosions were scored as 0 or 3 whereas hypoechogenicity and thickening were scored as 0 or 1 .

Statistical analysis. All analysis was done per entheseal site. Because there were some differences between the scoring methods, 2 types of analysis were performed, using the appropriate data:

- The presence and absence of each sonographic elementary lesion was compared with findings on PE at the same entheseal site, by using all 3 data sources because this was recorded by all.

- The weighted analysis including the scoring of the findings was performed using only the first 2 databases because scoring was done between 0 and 3 for all lesions.

The frequency of each elementary lesion on US was examined and presented as frequencies (percentages). The dependence between PE and US scores was assessed using chi-square or Fisher's exact tests, as appropriate. SPSS version 21 was used for analysis (IBM Corp.).

\section{RESULTS}

A total of 2298 entheses from 377 patients (341 with PsA, 36 with psoriasis) were compared using US and PE.

The presence of elementary lesions. Patients with clinical Achilles enthesitis had more frequent abnormalities on US (hypoechogenicity: $\mathrm{p}<0.001$, thickening: $\mathrm{p}=0.001$, Doppler positivity: $\mathrm{p}=0.002$, erosions: $\mathrm{p}=0.02$; Table 1 ). Similarly, hypoechogenicity $(\mathrm{p}=0.001)$ and enthesophytes/ calcifications $(\mathrm{p}=0.028)$ at the patellar tendon origin were more common in patients with clinical enthesitis and there was a tendency for more erosions but it did not reach statistical significance $(p=0.065)$. The clinical quadriceps enthesitis was related to hypoechogenicity on US $(p=0.001)$ and patients with clinical enthesitis on the lateral elbow had more frequent Doppler signals $(p=0.007)$. The rest of the entheseal sites were uncoupled from the US features, especially distal patellar tendon insertion and plantar aponeurosis (Table 1).

We have repeated the analysis by removing the patients with psoriasis. Focusing only on patients with PsA, the results were very similar to those of the whole group, with the exception of patellar tendon origin for calcifications (data not shown).

The severity of elementary lesions on US. For quadriceps tendon insertion, clinical enthesitis was linked to severity of hypoechogenicity $(\mathrm{p}=0.026)$ and calcifications $(\mathrm{p}=0.020)$ on US (Figure 1, and Supplementary Table, available with the online version of this article).

For patellar tendon origin, patients with clinical enthesitis had more severe hypoechogenicity $(\mathrm{p}<0.001)$, thickening $(p<0.001)$, enthesophytes $(p<0.001)$, and calcifications $(\mathrm{p}=0.003)$ on US.

For Achilles enthesitis, clinical enthesitis was associated with the severity of hypoechogenicity $(\mathrm{p}=0.008)$ and power Doppler $(\mathrm{p}=0.048)$ on US.

For common extensor tendon origin, the severity of hypoechogenicity $(\mathrm{p}=0.018)$ and power Doppler $(\mathrm{p}=0.017)$ was associated with clinical enthesitis (Figure 1, and Supplementary Table, available with the online version of this article).

\section{DISCUSSION}

Our findings confirm that the link between PE and US for enthesitis is dependent on the anatomical site. Patellar tendon origin and Achilles entheses are the 2 sites where PE is significantly linked to US findings, supporting the construct validity of US to visualize enthesitis.

The discrepancies between PE and US across different entheseal sites can be due to the difficulties in identifying the enthesis accurately by PE and different pain thresholds at different entheseal sites. For sites where PE is not linked to US, such as the plantar aponeurosis or the patellar tendon insertion, US may improve the assessment by eliminating the false positives and negatives of PE. However, there may also be technical difficulties in detecting some of the entheseal changes by US, such as the very low prevalence of Doppler signals in the plantar aponeurosis, or US not being capable of detecting osteitis. Our group has previously reported the relationship between each elementary lesion with PE findings directly at the entheseal insertions using US on 21 patients with SpA, for the enthesis around the knee only ${ }^{13}$. That study

Personal non-commercial use only. The Journal of Rheumatology Copyright @ 2020 . All rights reserved. 


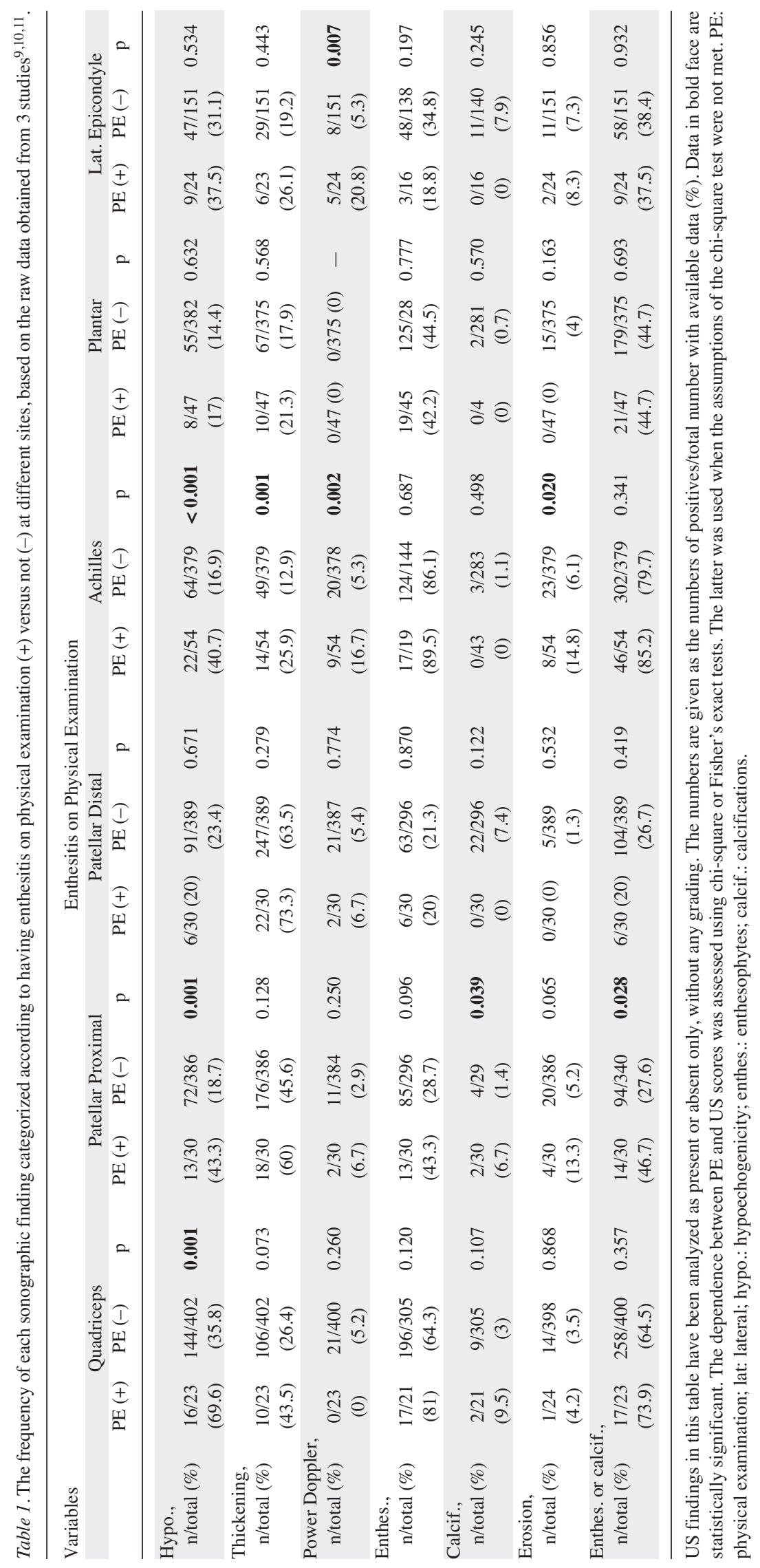

Personal non-commercial use only. The Journal of Rheumatology Copyright @ ${ }^{2020}$. All rights reserved. 


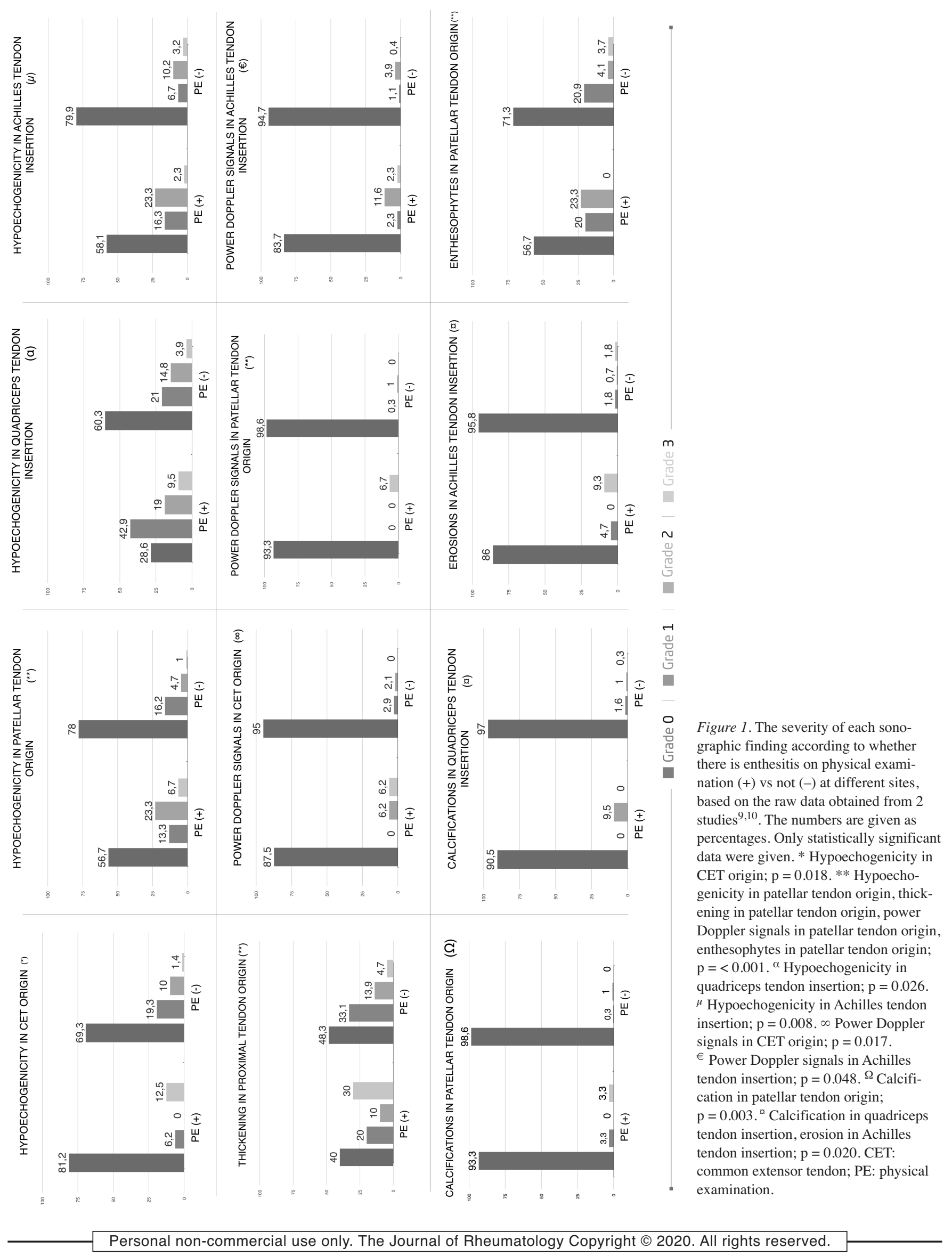


suggested that clinical enthesitis was associated with more hypoechogenicity and thickening on US. To the best of our knowledge, this is the first study on a large number of entheses and at multiple sites to examine the agreement between various sonographic features and PE to understand the clinical significance of individual lesions on US.

The enthesis is a very important structure in SpA, not only because it is frequently involved but also because of its significant effect on patients' pain, global assessment, and quality of life. The recognition of enthesitis is important both at diagnosis and at followup, to determine the most appropriate treatment. However, the enthesis is probably the most difficult musculoskeletal structure to assess because the same sites are commonly affected by mechanical tendinopathies/enthesopathies and owing to the proximity of fibromyalgia tender points ${ }^{14}$. It is important to accurately assess the cause of pain at the entheseal insertions to avoid overtreating or undertreating patients.

The major strengths of our study are the large number of entheses and the representation of 2 experienced sonographers in 3 different settings. Because the same scoring method was not applied, it was not possible to include all patients to link the severity of the US features with PE; but using the same definitions of elementary lesions, the presence/absence data were comparable. There were multiple clinical assessors for the third study, which may be considered a limitation. However, this perfectly reflects real-life experience and the assessors have been trained by the same individual.

The relationship between US and PE for enthesitis assessment depends on the entheseal site. US may be used to prove the presence of entheseal inflammation when there is diagnostic uncertainty or when disease activity is not clear and/or therapies are considered. In the absence of a gold standard histological method, these findings provide a platform for the assessment of clinically relevant enthesitis. Future research should aim to confirm these findings and further validate the currently existing clinical scoring systems for enthesitis in SpA.

\section{ONLINE SUPPLEMENT}

Supplementary material accompanies the online version of this article.

\section{REFERENCES}

1. McGonagle D, Khan MA, Marzo-Ortega H, O'Connor P, Gibbon W, Emery P. Enthesitis in spondyloarthropathy. Curr Opin Rheumatol 1999;11:244-50.

2. Kaeley GS, Eder L, Aydin SZ, Gutierrez M, Bakewell C. Enthesitis: a hallmark of psoriatic arthritis. Semin Arthritis Rheum 2018; 48:35-43.

3. Gandjbakhch F, Terslev L, Joshua F, Wakefield RJ, Naredo E, D'Agostino MA. Ultrasound in the evaluation of enthesitis: status and perspectives. Arthritis Res Ther 2011;13:188

4. Husic R, Gretler J, Felber A, Graninger WB, Duftner C, Hermann J, et al. Disparity between ultrasound and clinical findings in psoriatic arthritis. Ann Rheum Dis 2014;73:1529-36.

5. Bandinelli F, Prignano F, Bonciani D, Bartoli F, Collaku L, Candelieri A, et al. Ultrasound detects occult entheseal involvement in early psoriatic arthritis independently of clinical features and psoriasis severity. Clin Exp Rheumatol 2013;31:219-24.

6. Borman P, Koparal S, Babaoglu S, Bodur H. Ultrasound detection of entheseal insertions in the foot of patients with spondyloarthropathy. Clin Rheumatol 2006;25:373-7.

7. Balint PV, Kane D, Wilson H, McInnes IB, Sturrock RD. Ultrasonography of entheseal insertions in the lower limb in spondyloarthropathy. Ann Rheum Dis 2002;61:905-10.

8. Aydin SZ, Castillo-Gallego C, Ash ZR, Marzo-Ortega H, Emery P, Wakefield RJ, et al. Ultrasonographic assessment of nail in psoriatic disease shows a link between onychopathy and distal interphalangeal joint extensor tendon enthesopathy. Dermatology 2012;225:231-5.

9. Arslan Alhussain F, Kasapoglu Gunal E, Kurum E, Bakirci S, Ozturk AB, McGonagle D, et al. Greater magnitude of entheseal microdamage and repair in psoriatic arthritis compared to ankylosing spondylitis on ultrasound. Rheumatology 2019;58: 299-303.

10. Polachek A, Cook R, Chandran V, Gladman DD, Eder L. The association between sonographic enthesitis and radiographic damage in psoriatic arthritis. Arthritis Res Ther 2017;19:189.

11. Terslev L, Naredo E, Iagnocco A, Balint PV, Wakefield RJ, Aegerter $\mathrm{P}$, et al. Defining enthesitis in spondyloarthritis by ultrasound: results of a Delphi process and of a reliability reading exercise. Arthritis Care Res 2014;66:741-8.

12. de Miguel E, Cobo T, Munoz-Fernandez S, Naredo E, Uson J, Acebes JC, et al. Validity of enthesis ultrasound assessment in spondyloarthropathy. Ann Rheum Dis 2009;68:169-74.

13. Aydin SZ, Tan AL, Hodsgon R, Grainger A, Emery P, Wakefield RJ, et al. Comparison of ultrasonography and magnetic resonance imaging for the assessment of clinically defined knee enthesitis in spondyloarthritis. Clin Exp Rheumatol 2013;31:933-6.

14. Roussou E, Ciurtin C. Clinical overlap between fibromyalgia tender points and enthesitis sites in patients with spondyloarthritis who present with inflammatory back pain. Clin Exp Rheumatol 2012;30:24-30. 